International Journal of Pure and Applied Mathematics

Volume 111 No. 2 2016, 219-231

ISSN: 1311-8080 (printed version); ISSN: 1314-3395 (on-line version)

url: http://www.ijpam.eu

doi: $10.12732 /$ ijpam.v111i2.8

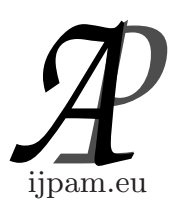

\title{
MATHEMATICAL ANALYSIS OF A CHOLERA TRANSMISSION MODEL INCORPORATING MEDIA COVERAGE
}

\author{
Musundi O. Beryl ${ }^{1 \S}$, Lawi O. George ${ }^{2}$, Nyamwala O. Fredrick ${ }^{3}$ \\ ${ }^{1,2}$ Department of Mathematics and Physics \\ Moi University \\ P.O. Box 3900, Eldoret, KENYA \\ ${ }^{2}$ Department of Mathematics \\ Masinde Muliro University of Science and Technology \\ P.O. Box 190, Kakamega, KENYA
}

\begin{abstract}
Diarrhoeal diseases are the major cause of child mortality in developing countries, where access to clean drinking water and sanitation is a problem. In this paper, we develop and analyse a mathematical model for cholera transmission incorporating media coverage. The existence and stability of the equilibrium points is established. Analysis of the model shows that the disease free equilibrium is both locally and globally asymptotically stable when the basic reproduction number is less than unity while the endemic equilibrium is locally asymptotically stable when the reproduction number is greater than unity. Numerical simulations done using the MATLAB software indicate that when media coverage is very efficient, the number of cholera infectives decreases faster, impliying that media alert and awareness campaigns are vital in controlling the spread of cholera.
\end{abstract}

AMS Subject Classification: 92B05

Key Words: mathematical model, cholera, media coverage, stability, basic reproduction number

Received: $\quad$ August 22, 2016

Revised: $\quad$ October 5, 2016

Published: December 11, 2016

$\S_{\text {Correspondence author }}$ (c) 2016 Academic Publications, Ltd. url: www.acadpubl.eu 


\section{Introduction}

Cholera is a gastrointestinal disease caused by the bacterium Vibrio cholerae. It affects millions of people worldwide, with an estimated $1.4-4.3$ million cases and 28,000 - 142, 000 deaths reported per year [6]. The disease dynamics depend mainly on the interactions between the human host, the pathogen and the environment [5], which leads to two transmission pathways that is the human to human and the environment to human transmission.

Cholera is mostly common in developing countries like Africa, parts of Asia and South and Central America where there is inadequate sanitation and lack of clean drinking water. In 2012, 94,553 cholera cases with 1, 834 associated deaths were reported to the WHO by 25 African countries [1]. In Kenya, for instance, 14, 878 cholera cases with 234 deaths were recorded between December 2014 and May 2016 [12].

Mathematical modelling is an important tool used in analysing the dynamics of infectious diseases. Several models have been formulated and analysed to explain the dynamics of cholera transmission. Codeço [8], proposed a model that looked at the role of the aquatic reservoir in the maintenance of endemic cholera. Hartley et al. [9], modified Codeço's model to include a hyperinfectious state of the bacterium based on laboratory observations. Mukandavire et al. [4], simplified the model by Hartley et al. [9], to study the 2008-2009 cholera outbreak in Zimbabwe. In his model, he explored the "fast" human-to-human and "slow" environment-to-human transmission modes of cholera. His results showed that both modes of transmission contributed in sustaining cholera in Zimbabwe. Wang et al. [11], extended the model by Mukandavire et al. [4], to include the effects of vaccination, therapeutic treatment and water sanitation . Numerical simulation of his results shows that the various control measures are closely interrelated and that the strength of one measure as an optimal strategy depends on its relative cost and the population setting.

Media coverage has been known to greatly influence an individuals behaviour as well as goverment policies on prevention and control of infectious diseases [7]. In this paper, we seek to understand the effects of media coverage in the transmission of cholera. We extend the model proposed by Mukandavire et al. [4], to include the effects of media coverage. The rest of the paper is organized as follows: the model is formulated in Section 2, existence and stability analysis of the disease free equilibrium is done in Section 3, existence and stability analysis of the endemic equilibrium is done in Section 4 numerical simulations are carried out in Section 5. The paper ends with a conclusion in Section 6 . 


\section{Model Formulation and Description}

The human population is subdivided into classes of susceptible, $S$, infected , $I$ and recovered, $R$ with the total population $N(t)=S(t)+I(t)+R(t)$. $B$ denotes the concentration of Vibrios in the environment. As in [4], susceptible individuals acquire Vibrios from ingesting environmental Vibrios or through human to human transmission. Therefore, we obtain a model represented by the following system of equations

$$
\begin{aligned}
\frac{d S}{d t} & =\Lambda-\left(\beta_{e}-\rho \beta_{e}\right) \frac{S B}{\kappa+B}-\left(\beta_{h}-\frac{\rho \beta_{h} I}{m+I}\right) S I-\mu S \\
\frac{d I}{d t} & =\left(\beta_{e}-\rho \beta_{e}\right) \frac{S B}{\kappa+B}+\left(\beta_{h}-\frac{\rho \beta_{h} I}{m+I}\right) S I-(\gamma+\mu+\delta) I \\
\frac{d B}{d t} & =\sigma I-\xi B \\
\frac{d R}{d t} & =\gamma I-\mu R
\end{aligned}
$$

where $\mu$ denotes the natural death rate, $\Lambda$ is the rate at which individuals are recruited into the population, $\beta_{e}$ is the rate of ingestion of Vibrios from the environment, $\rho \beta_{e}(0<\rho<1)$ is the reduced rate of ingestion of Vibrios from the environment due to media coverage, where $\rho$ measures the efficacy of media coverage, $\beta_{h}$ is the rate of human to human transmission, $\frac{\rho \beta_{\mathrm{h}} I}{m+I}$ is the reduced rate of contact with infected persons due to media alert where the function $\frac{I}{m+I}$ is a continuous bounded function which takes into account disease saturation or psychological effects, $\kappa$ is the pathogen concentration that yields $50 \%$ chance of catching cholera, $\gamma$ is the rate of recovery, $\delta$ is the death rate due to infection, $m$ is the rate of media coverage, $\sigma$ is the rate of human contribution to Vibrio cholerae and $\xi$ is the death rate of Vibrio cholerae.

Since $R(t)=N(t)-S(t)-I(t)$, it is enough to consider the first three equations of system 1 , our new system becomes

$$
\begin{aligned}
\frac{d S}{d t} & =\Lambda-\left(\beta_{e}-\rho \beta_{e}\right) \frac{S B}{\kappa+B}-\left(\beta_{h}-\frac{\rho \beta_{h} I}{m+I}\right) S I-\mu S \\
\frac{d I}{d t} & =\left(\beta_{e}-\rho \beta_{e}\right) \frac{S B}{\kappa+B}+\left(\beta_{h}-\frac{\rho \beta_{h} I}{m+I}\right) S I-(\gamma+\mu+\delta) I \\
\frac{d B}{d t} & =\sigma I-\xi B
\end{aligned}
$$

The model monitors a population, therefore, solutions with positive initial data, will remain positive for $t \geq 0$ and will be bounded in the feasible region $\Omega=$ $\left\{(S, I, R): N \leq \frac{\Lambda}{\mu}\right\}$ 


\section{Disease Free Equilibrium}

The disease-free equilibrium $\left(E^{0}\right)$ is a point where the disease is not present in the population. The disease free equilibrium (DFE) of the system $\left(E^{0}\right)$ is given by $\left(\frac{\Lambda}{\mu}, 0,0\right)$. We must first find the basic reproduction number inorder to analyse the DFE.

\subsection{Basic Reproduction Number}

The basic reproduction number $R_{0}$ is the average number of secondary infections caused when a single infectious individual is introduced into a purely susceptible population. We determine $R_{0}$ using the next generation matrix approach [3]. The basic reproduction number is the spectral radius $\rho F V^{-1}$ which is given by

$$
R_{0}=\frac{\Lambda}{\mu(\gamma+\delta+\mu)}\left(\beta_{h}+\frac{\left(\beta_{e}-\rho \beta_{e}\right) \sigma}{\kappa \xi}\right)
$$

\subsection{Local Stability of the Disease Free Equilibrium}

Theorem 1. The disease free equilibrium is locally asymptotically stable if $R_{0}<1$ and is unstable if $R_{0}>1$.

Proof. The jacobian of system at the DFE is given by.

$$
J=\left[\begin{array}{ccc}
-\mu & -\beta_{h} \frac{\Lambda}{\mu} & -\left(\beta_{e}-\rho \beta_{e}\right) \frac{\Lambda}{\mu \kappa} \\
0 & \beta_{h} \frac{\Lambda}{\mu}-(\gamma+\mu+\delta) & \left(\beta_{e}-\rho \beta_{e}\right) \frac{\Lambda}{\mu \kappa} \\
0 & \sigma & -\xi
\end{array}\right]
$$

Clearly one of the eigen values is $-\mu$, we use the Routh Hurwitz criterion [2] to check the signs of the eigen values of the reduced block matrix given by

$$
\left[\begin{array}{cc}
\beta_{h} \frac{\Lambda}{\mu}-(\gamma+\mu+\delta) & \left(\beta_{e}-\rho \beta_{e}\right) \frac{\Lambda}{\mu \kappa} \\
\sigma & -\xi
\end{array}\right]
$$

The determinant is given by

$$
-\xi\left[\beta_{h} \frac{\Lambda}{\mu}-(\gamma+\mu+\delta)\right]-\frac{\sigma A \Lambda}{\mu \kappa}
$$

where $\left(\beta_{e}-\rho \beta_{e}\right)=A$. For the determinant to be positive

$$
\xi \beta_{h} \frac{\Lambda}{\mu}+\frac{\sigma A \Lambda}{\mu \kappa}<\xi(\gamma+\mu+\delta)
$$


dividing both sides by $\xi \gamma+\mu+\delta)$ gives

$$
\frac{\Lambda}{\mu(\gamma+\delta+\mu)}\left[\beta_{h}+\frac{\left(\beta_{e}-\rho \beta_{e}\right) \sigma}{\kappa \xi}\right]<1
$$

Thus $R_{o}<1$. The trace of the reduced block matrix is given by

$$
\beta_{h} \frac{\Lambda}{\mu}-\xi-(\gamma+\mu+\delta)
$$

If we make $\gamma+\mu+\delta$ to be the subject of the formula and $\left(\beta_{e}-\rho \beta_{e}\right)=A$ from the basic reproduction number, and subsitute it in the trace, we get

$$
\beta_{h} \frac{\Lambda}{\mu}-\left[\frac{\Lambda}{\mu R_{0}}\left(\beta_{h}+\frac{A \sigma}{\kappa \xi}\right)\right]-\xi
$$

Equation (4) needs to be negative for us to get negative eigen values, since all the other parameters are negative, we find conditions that make Equation (5) negative

$$
\beta_{h} \frac{\Lambda}{\mu}-\frac{\Lambda}{\mu R_{0}} \beta_{h}
$$

This is only true if $R_{o}<1$, and therefore the disease free equilibrium is locally asymptotically stable.

\subsection{Global Stability of Disease Free Equilibrium}

We use the Castillo-Chavez theorem [10] to investigate the global asymptotic stability of the disease free state. We therefore rewrite system (2) in the form

$$
\begin{aligned}
& \frac{d X}{d t}=H(X, Z) \\
& \frac{d Z}{d t}=G(X, Z), G(X, 0)=0
\end{aligned}
$$

where $X=(S)$ and $Z=(I, B)$. Here the components of $X \in \mathbb{R}$ denote the uninfected individuals and the components of $Z \in \mathbb{R}$ denote the infected individuals. The disease free equilibrium of the system now becomes $E^{0}=$ $\left(X^{*}, 0\right), X^{*}=\left(\frac{\Lambda}{\mu}\right)$. To guarantee local asymptotic stability, the following two conditions must be met.

1. $\frac{d X}{d t}=H(X, 0), X^{*}$ is globally asymptotically stable(GAS)

2. $G(X, Z)=P Z-\widehat{G}(X, Z), \widehat{G}(X, Z) \geq 0$ for $(X, Z) \in \Omega$ 
where $P=D_{Z} G\left(X^{*}, 0\right)$ is an $M$ matrix (the off diagonal elements of $P$ are nonnegative) and $\Omega$ is the region where the model makes biological sense. If system (2) satisfies conditions 1 and 2 then the following theorem holds.

Theorem 2. The fixed point $E^{0}=\left(X^{*}, 0\right)$ is a globally asymptotic stable equilibrium of the system (2) provided that $R_{0}<1$ and the assumptions 1 and 2 are satisfied.

Proof. Since $X=(S)$ and $Z=(I, B)$, then

$$
\begin{gathered}
H(X, 0)=[\Lambda-\mu S] \\
G(X, Z)==P Z-\widehat{G}(X, Z)
\end{gathered}
$$

where

$$
P=\left[\begin{array}{cc}
\beta_{h} S-(\gamma+\mu+\delta) & \left(\beta_{e}-\rho \beta_{e}\right) \frac{S}{\kappa} \\
\sigma & -\xi
\end{array}\right]
$$

and

$$
\widehat{G}(X, Z)=\left[\begin{array}{c}
\left(\frac{\rho \beta_{\mathrm{h}} I}{m+I}\right) S I+\left(\beta_{e}-\rho \beta_{e}\right) \frac{S B^{2}}{\kappa(\kappa+B)} \\
0
\end{array}\right]
$$

Since $0<\rho<1$, then $\widehat{G}(X, Z) \geq 0$. The conditions 1 and 2 have been met and therefore $E^{0}$ is globally asymptotically stable.

\section{Endemic Equilibrium}

This a point where the disease spreads in the population. We denote our endemic equilibrium point as $E^{*}=\left(S^{*}, I^{*}, B^{*}\right)$.

Theorem 3. There exists a unique endemic equilibrium of system (2) when $R_{0}>1$.

Proof. We equate the right hand side of system (2) to zero to get.

$$
\begin{gathered}
B^{*}=\frac{\sigma}{\xi} I^{*} \\
S^{*}=\frac{\Lambda}{\mu}-\frac{(\gamma+\mu+\delta) I^{*}}{\mu}
\end{gathered}
$$


Substituting Equation (6) and (7) in second equation of system (2) gives us $I^{*}=0$ and

$$
A I^{* 3}+B I^{* 2}+C I^{*}+D=0
$$

where

$$
\begin{gathered}
A=(\rho-1)(\gamma+\mu+\delta) \beta_{h} \sigma, \\
B=(1-\rho) \beta_{h} \Lambda \sigma-(\gamma+\mu+\delta)\left[\left(\beta_{e}-\rho \beta_{e}\right) \sigma+m \beta_{h} \sigma+\beta_{h} \kappa \xi-\rho \beta_{h} \kappa \xi+\mu \sigma\right], \\
C=\left(\beta_{e}-\rho \beta_{e}\right) \Lambda \sigma+[m \Lambda \sigma+\Lambda \kappa \xi-\rho \Lambda \kappa \xi] \beta_{h} \\
-(\gamma+\mu+\delta)\left[m\left(\beta_{e}-\rho \beta_{e}\right) \sigma+m \beta_{h} \kappa \xi+m \mu \sigma+\mu \kappa \xi\right], \\
D=m\left(\beta_{e}-\rho \beta_{e}\right) \Lambda \sigma+m \beta_{h} \Lambda \kappa \xi-m(\gamma+\mu+\delta) \mu \kappa \xi .
\end{gathered}
$$

The endemic equilibrium of the system exists if the roots of Equation (8) are real and positive. We use the Descartes rule of signs to determine if positive real roots exist. Since the sign of $\mathrm{A}$ is negative and $\mathrm{D}$ is positive when $R_{0}>1$, there exists atleast one positive real root hence the endemic equilibrium exists.

Theorem 4. The endemic equilibrium ( $\left.E^{*}\right)$ of system (2) is locally asymptotically stable when $R_{0}>1$

Proof. The jacobian matrix evaluated at the endemic equilibrium point is given by

$$
J\left(E^{*}\right)=\left[\begin{array}{ccc}
-X-\mu & -Y & -Z \\
X & Y-(\gamma+\mu+\delta) & Z \\
0 & \sigma & -\xi
\end{array}\right]
$$

where

$$
\begin{aligned}
X & =\frac{\left(\beta_{e}-\rho \beta_{e}\right) B^{*}}{\kappa+B^{*}}+\left(\beta_{h}-\frac{\rho \beta_{h} I^{*}}{m+I^{*}}\right) I^{*} \\
Y & =\left[\beta_{h}-\frac{\rho \beta_{h} I^{*}\left(2 m+I^{*}\right)}{\left(m+I^{*}\right)^{2}}\right] S^{*} \\
Z & =\frac{\left(\beta_{e}-\rho \beta_{e}\right) s^{*} \kappa}{\left(\kappa+B^{*}\right)^{2}}
\end{aligned}
$$

The characteristic equation of the matrix will be

$$
a_{0} \lambda^{3}+a_{1} \lambda^{2}+a_{2} \lambda+a_{3}=0
$$


where

$$
\begin{aligned}
& a_{0}=1 \\
& a_{1}=-Y+(\gamma+\mu+\delta)+\xi+(X+\mu) \\
& a_{2}=(X+\mu)[-Y+(\gamma+\mu+\delta)+\xi]-Y \xi+(\gamma+\mu+\delta) \xi-\sigma Z+X Y \\
& a_{3}=(X+\mu)[-Y \xi+(\gamma+\mu+\delta) \xi-\sigma Z]-X \sigma Z+X Y \xi
\end{aligned}
$$

For the Routh Hurwitz criterion [2] to be satisfied, $a_{1}>0, a_{2}>0, a_{3}>0$ and $a_{1} a_{2}-a_{3}>0$. At the endemic equilibrium the right hand side of the second and third equation of system (2) becomes zero giving us

$$
(\gamma+\mu+\delta)=\left(\beta_{e}-\rho \beta_{e}\right) \frac{S^{*} \sigma}{\kappa \xi+\sigma I^{*}}+\left(\beta_{h}-\frac{\rho \beta_{h} I^{*}}{m+I^{*}}\right) S^{*}
$$

From Equation(9) we can easily show that

$$
\begin{gathered}
-Y+(\gamma+\mu+\delta)+\xi>0 \\
-Y \xi+(\gamma+\mu+\delta) \xi-\sigma Z>0
\end{gathered}
$$

Since all the other parameters are positive and equation (10) and (11) are also positive, the conditions [2] hold and therefore the endemic equilibrium is locally asymptotically stable.

\section{Numerical Simulation}

We carry out numerical simulations using the MATLAB software to illustrate the behaviour of our system. The parameter values are given in Table 1. From Equation (3) and when $\beta_{h}=5$ we compute $R_{0}=3.794>1$.

When $R_{0}>1$, the cholera free equilibrium becomes unstable and the endemic equilibrium becomes stable. Consequently, the endemic equilibrium is asymptotically stable. Figure 1 shows that all solutions of $\mathrm{S}(\mathrm{t})$ and $\mathrm{I}(\mathrm{t})$ converge to $E^{*}$ when $R_{0}>1$ supporting Theorem 4 .

Lack of media coverage about an outbreak of the disease causes the number of infectives to first rise steadily as many people are not yet aware about the outbreak and the preventive measures before it starts droping as the susceptibles are depleted as shown in Figure 2.

In the presence of the media coverage the number of infectives decreases sharply as many people are aware of the outbreak and take precautionary measures to prevent infection as shown in Figure 3. 


\begin{tabular}{|c|c|c|}
\hline Parameter & Value & Source \\
\hline$\Lambda$ & $9.6274 \times 10^{-5} /$ day & {$[13]$} \\
\hline$\mu$ & $2.537 \times 10^{-5} /$ day & {$[13]$} \\
\hline$\beta_{e}$ & $0.75 /$ day & Estimate \\
\hline$\beta_{h}$ & $1 /$ day & Varies \\
\hline$\kappa$ & $10^{6}$ cells $/$ ml & {$[8]$} \\
\hline$\gamma$ & $5 p e o p l e /$ day & {$[9]$} \\
\hline$\delta$ & $4.0 \times 10^{-4} /$ day & {$[14]$} \\
\hline$m$ & 0.00001 & Varies \\
\hline$\sigma$ & 10 cells $/$ ml-day & {$[11]$} \\
\hline$\xi$ & $0.23 /$ day & {$[14]$} \\
\hline$\rho$ & 0.6 & Varies \\
\hline
\end{tabular}

Table 1: Model Parameters and Values

When $R_{0}<1$ and the media is very effective in reporting about the outbreak of the disease and the preventive measures, the infectious individuals are eliminated faster from the population, while if there is ineffective or no media coverage then the infection spreads for a longer time in the population as shown in Figure 4. 


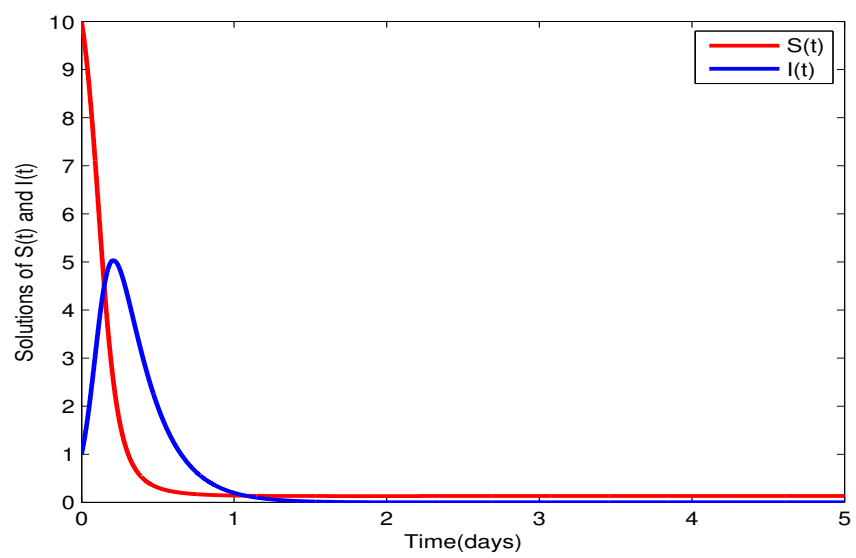

Figure 1: Numerical solutions when $R_{0}>1$

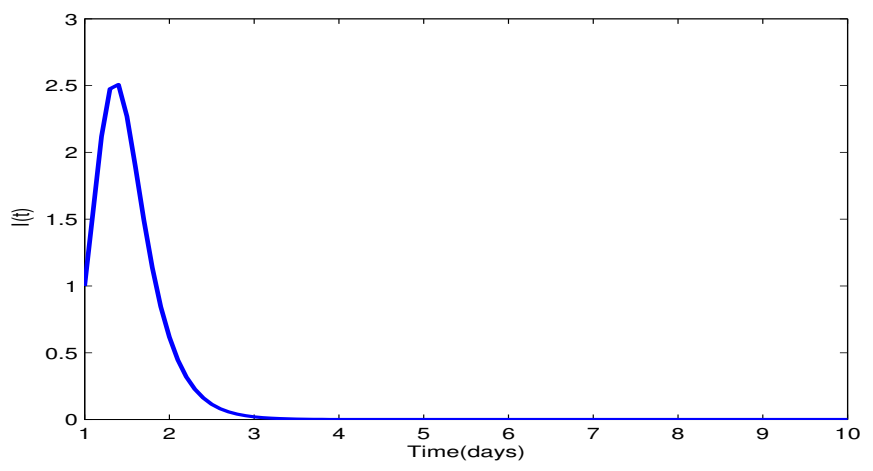

Figure 2: Cholera infectives in the absence of media coverage $(m=0$ and $\rho=0$ ) 


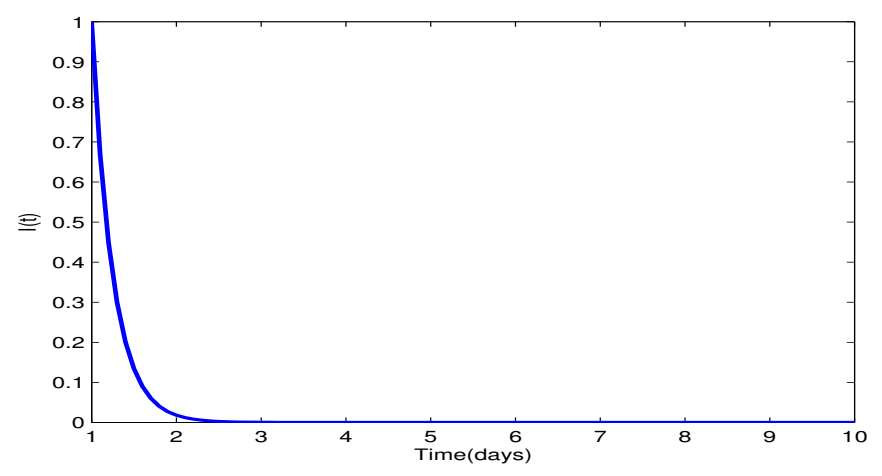

Figure 3: Cholera infectives in the presence of media coverage $(m=$ 0.00001 and $\rho=0.6$ )

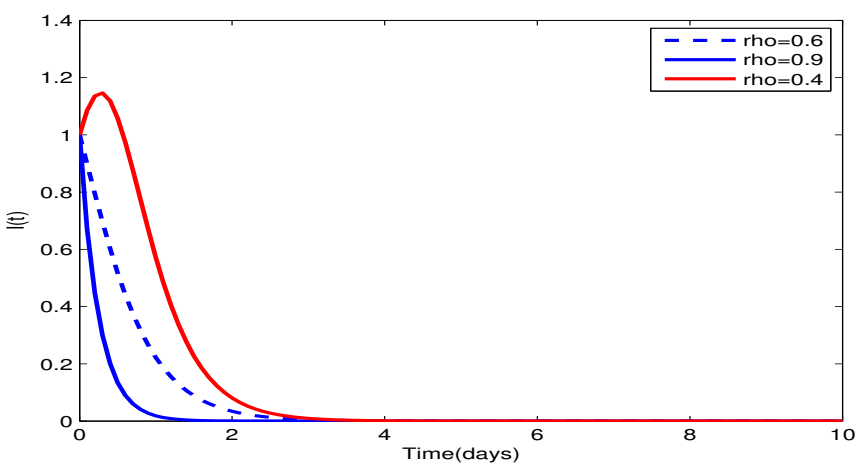

Figure 4: Cholera infectives with different values of $\rho$ 


\section{Conclusion}

In this paper, we formulated a mathematical model for cholera transmission incorporating media coverage. We studied the stability of the disease free and endemic equilibrium. The results of the disease free equilibrium showed that the model is both locally and globally stable when $R_{0}<1$, thus reducing $R_{0}$ to less than unity reduces the disease spread. Next we studied the endemic equilibrium which we found to be asymptotically stable when $R_{0}>1$. Numerical analysis shows that in the presence of media coverage the disease dies out faster while lack of effective media reporting on the presence of the disease and preventive measures greatly increases the number of infectious people in the population which is not favourable for the eradication of the disease. The model has not carried out optimal control and cost effectiveness of different cholera intervention strategies, which can be explored in future to find out which strategy is the best in the control of the disease

\section{Acknowledgements}

The authors would like to acknowledge the African Mathematics Millenium Science initiative (AMMSI) and the National Commission for Science, Technology and Innovation (NACOSTI) for the generous financial support through their grants.

\section{References}

[1] E. Mintz and R. Tauxe, Cholera in Africa: A closer look and a time for action, Journal of Infectious Diseases, 208, 1 (2013), 4-7. DOI:10.1093/infdis/jit205.

[2] M. Li and L. Wang, A criterion for stability of matrices, Journal of Mathematical Analysis and Applications, 225, 1 (1998), 249-264. DOI:10.1006/jmaa.1998.6020.

[3] P. Van den Driessche and J. Watmough, Reproduction numbers and sub-threshold endemic equilibria for compartmental models of disease transmission, Mathematical Biosciences, 180, 1-2 (2002), 29-48. DOI:10.1016/s0025-5564(02)00108-6.

[4] Z. Mukandavire, S. Liao, J. Wang, H. Gaff, D. Smith, and Morris, Estimating the reproductive numbers for the 2008 - 2009 cholera outbreaks in Zimbabwe, Proceedings of the National Academy of Sciences, 108, 21 (2011), 8767-8772. DOI:10.1073/pnas.1019712108.

[5] E. Nelson, J. Harris, J. Morris, S. Calderwood and A. Camilli, Cholera transmission: the host, pathogen and bacteriophage dynamics, Nature Reviews: Microbiology, 7, 10 (2009), 693-702. DOI:10.1038/nrmicro2204. 
[6] WHO media center, Cholera factsheet N107, Available from www.who.int/mediacentre/factsheets/fs107/en/

[7] A. Misra, A. Sharma and J. Shukla, Modeling and analysis of the effects of awareness programs by media on the spread of infectious diseases, Mathematical and computer modelling, 53, 5-6 (2011), 1221-1228. DOI:10.1016/j.mcm.2010.12.005.

[8] C. Codeço, Endemic and epidemic dynamics of cholera: the role of the aquatic reservoir, BMC Infectious diseases, 1, 1 (2001). DOI:10.1186/1471-2334-1-1.

[9] D. Hartley, J. Morris and D. Smith, Hyperinfectivity: A critical element in the ability of V. cholerae to cause epidemics?, Plos Medicine, 3, 1 (2005), 0063-0069. DOI:10.1371/journal.pmed.0030007.

[10] C. Castillo-Chavez, Z. Feng and W. Huang, On the computation of $R_{0}$ and its role on global stability, Mathematical Approaches for Emerging and Reemerging Infectious Diseases, 125, (2002), 229-250. DOI:10.1007/978-1-4757-3667-0-13.

[11] J. Wang and C. Modnak, Modeling cholera dynamics with controls, Canadian Applied Mathematics Quarterly, 19, 3 (2011), 255-273.

[12] United Nations Children's Emergency Fund. Kenya: Cholera outbreak overview. Available from www.unicef.org:60090.

[13] G. Lawi, J. Mugisha and N. Omolo-Ongati, Mathematical model for malaria meningitis co-infection among children, Applied Mathematical Sciences, 5, 47 (2011), 2337-2359.

[14] L. Mari, E. Bertuzzo, L. Righetto, R. Casagrandi, M. Gatto, I. Rodriquez-Iturbe and A. Rinaldo, Modelling cholera epidemics: the role of waterways, human mobility and sanitation, Journal of the Royal Society Interface, (2011). DOI:10.1098/rsif.2011.0304. 
